\title{
Cyclotron Emission Features in the Spectra of Polars with Low Accretion Rates
}

\author{
E. D. Gospodchikov
}

Higher School of General 6 Applied Physics, Nizhny Novgorod State University, 23 Gagarin prosp., 603950 Nizhny Novgorod, Russia

\author{
A. V. Serber \\ Institute of Applied Physics of the Russian Academy of Sciences, \\ 46 Ulyanov st., 603950 Nizhny Novgorod, Russia
}

\begin{abstract}
We calculate non-LTE transfer of cyclotron-harmonics radiation in the hot spot of an accreting magnetic white dwarf (WD) in the case where the contribution of the accretion column to the observed radiation is negligible. The observed cyclotron spectra of a polar with a low accretion rate are found. Based on the theory developed, we interpret the cyclotron emission line spectra of the systems and determine physical conditions in the emission regions of HS $1023+3900$ and $\mathrm{HS} 0922+1333$.
\end{abstract}

\section{Introduction}

Phase resolved spectroscopy of low accretion rate polars HS $1023+3900$ (Reimers, Hagen, \& Hopp 1999) and HS 0922+1333 (Reimers \& Hagen 2000) revealed variable patterns of cyclotron emission features at harmonics with numbers $s$ from 2 to 4 . The main and secondary accretion poles in HS $1023+3900$ have magnetic fields $B=60$ and $68 \mathrm{MG}$, respectively, and show such features at $s=2$ and 3 . The intensities of the main-pole 2 nd and 3rd harmonics and the secondary-pole 2nd harmonic have a simple time dependence with one maximum per orbital period. The intensity of the secondary-pole 3rd harmonic as a function of orbital phase has two maxima and a minimum in between. The latter minimum appears at the time when the second harmonic from this pole is maximum. In the phase-resolved spectra of HS 0922+1333, cyclotron emission from the main pole with $B=66 \mathrm{MG}$ is clearly seen at $s=2-4$. In this case, the temporal dependences of $2 \mathrm{nd}$ and 3rd harmonics show one maximum, while the 4th harmonic as a function of time has two maxima. The minimum between these maxima appears simultaneously with the maxima of the 2nd and 3rd harmonics. The much weaker 2 nd and 3 rd harmonics from the secondary pole correspond to $B=81 \mathrm{MG}$ and appear in the antiphase with the main-pole cyclotron emission.

Cyclotron spectra of accreting regions in polars and CVs are conventionally interpreted in terms of thermal cyclotron emission formed under LTE conditions. In fact, this means that the electron distribution in the source is assumed isotropic Maxwellian with a given temperature $T$, so that the Kirchhoff law $\eta_{s l}=\chi_{s l} B_{\omega}(\omega)$, where $B_{\omega}(\omega)$ is the Planck intensity per one mode, holds for the 
cyclotron emissivity $\eta_{s l}$ and opacity $\chi_{s l}$ at the $s$ th harmonic for mode $l$ (hereafter, $l=1$ and 2 denote the extraordinary and ordinary mode, respectively). In particular, such an LTE model was used by Schwope et al. (2002) for an interpretation of the cyclotron spectra of HS $1023+3900$ and HS $0922+1333$.

As a rule, the assumption on LTE is taken for granted without special justification. However, it is only valid if $\varepsilon \gg 1$ or, at least, $\varepsilon \geq 1$. Here, $\varepsilon$ is the ratio between the time $t_{c} \simeq 3 m c^{3} /\left(4 e^{2} \omega_{B}^{2}\right)$ of transverse-energy loss of an electron due to cyclotron radiation and the time $\nu_{\text {eff }}^{-1}$ between collisions,

$$
\nu_{\mathrm{eff}}=8 \sqrt{2 \pi}(1+1 / \sqrt{2}) e^{4} N L /\left(15 m^{2} v_{T}^{3}\right)
$$

is the effective collision rate, $L=\ln \left[m v_{T}^{2} / \hbar \omega_{B}\right]$ is the magnetic Coulomb logarithm, $N$ is the electron density, $v_{T}=\beta_{T} c=(\kappa T / m)^{1 / 2}$ is the electron thermal velocity, $\omega_{B}=e B /(m c)$ is the electron gyrofrequency, $e$ and $m$ are the electron charge and mass, respectively, and $c$ is the speed of light. Thus, LTE conditions for cyclotron radiation can be realized only if $N$ is high enough. For example, if $T=10^{7} \mathrm{~K}$ and $B=60 \mathrm{MG}$, then the parameter $\varepsilon$ is equal to unity for $N \simeq 2.5 \times 10^{16} \mathrm{~cm}^{-3}$.

Taking into account that LTE may not always be realized for cyclotron emission from hot spots of accreting white dwarfs and that it is not so easy to check a priori the validity of the LTE assumption, we apply a non-LTE model of cyclotron radiation transfer for the interpretation of the phase-resolved cyclotron spectra of HS $1023+3900$ and HS $0922+1333$.

\subsection{Model geometry}

An accretion pole is modelled by a circular hot plasma slab with radius $r$ and height $H \ll r$, located on the surface of a white dwarf with radius $R_{*} \gg r$, which rotates synchronously together with its binary companion. The electron density $N$ and the temperature $T_{\|}$along the magnetic field $\mathbf{B}$ are assumed constant. The slab height $H$ is much larger than the size $l_{B} \simeq \beta_{T_{\|}} L_{B}$ of a gyroresonance layer in a magnetic field with the characteristic scale $L_{B}$ (here, $\left.\beta_{T_{\|}}=\left(\kappa T_{\|} / m c^{2}\right)^{1 / 2}\right)$, so that the vertical nonuniformity of the magnetic field is negligible. We allow for the possible variations in $B$ over the slab area, which, together with the thermal motion of electrons along the magnetic field, can contribute to the observed widths of the cyclotron spectral features. The magnetic-field direction is assumed vertical, and variations in the direction of $\mathbf{B}$ over the slab are neglected.

\section{Non-LTE transfer of cyclotron radiation}

Transfer of cyclotron radiation in a slab modeling an accretion pole is described by the equations

$$
\cos \alpha \frac{\partial I_{s l}(z, \xi, \alpha)}{\partial z}=-\chi_{s l}(z, \xi, \alpha) I_{s l}(z, \xi, \alpha)+\eta_{s l}(z, \xi, \alpha)
$$

Here, $\xi=p_{\|} / \sqrt{2} m c \beta_{T_{\|}}$is the dimensionless frequency variable,

$$
I_{s l}(z, \xi, \alpha)=\int I_{\omega}(z, \omega, \alpha) \delta\left[\omega\left(1-\sqrt{2} \beta_{T_{\|}} \xi \cos \alpha\right)-s \omega_{B}\right] \mathrm{d} \omega
$$


is the specific intensity in the mode $l$ at the $s$ th harmonic, which interacts with electrons whose longitudinal momentum is equal to $p_{\|}, \alpha$ is the angle between the the wave vector and the magnetic field, and the delta-function accounts for the Doppler effect related to the thermal motion of electrons along the magnetic field. If the recoil effect and natural line broadening are neglected, then the radiation frequency $\omega$ and the angle $\alpha$ are always related, via the Doppler formula $p_{\|}=m c\left(\omega-s \omega_{B}\right) /(\omega \cos \alpha)$, to the same longitudinal momentum $p_{\|}$of the electrons interacting with this radiation by virtue of cyclotron radiative processes. Thus, the radiation corresponding to some $p_{\| \mid}$is transferred independently of the radiation corresponding to other values of $p_{\|}$(Zheleznyakov \& Litvinchuk 1987; Zheleznyakov 1996).

According to the method of Einstein coefficients, cyclotron emissivity and opacity are determined by the electron distribution function and the probability of radiative transitions of electrons between the Landau levels (Zheleznyakov 1983, 1996; Serber 1990):

$$
\begin{gathered}
\eta_{s l}=\frac{\hbar \omega_{B}^{3} s^{3}}{8 \pi^{3} c^{2}} F_{s l} \sum_{j=s}^{\infty} \frac{j ! N_{j}}{s !(j-s) !}, \quad \chi_{s l}=F_{s l} \sum_{j=s}^{\infty} \frac{j !\left(N_{j-s}-N_{j}\right)}{s !(j-s) !} \\
F_{s l}=\frac{\sqrt{2} \pi^{2} e^{2}}{m c \beta_{T_{\|}} \omega_{B}} \frac{b_{s l}(\alpha) \phi(\xi)}{s !|\cos \alpha|}\left(\frac{\hbar \omega_{B} \sin ^{2} \alpha}{2 m c^{2}}\right)^{s-1}
\end{gathered}
$$

where $N_{j}$ is the population of the $j$ th Landau level $(j \geq 0), \sum_{j=0}^{\infty} N_{j}=N$, the function $\phi(\xi)=\pi^{-1 / 2} e^{-\xi^{2}}$ describes the Doppler profile of the cyclotron line, and the polarization factors

$$
\begin{gathered}
b_{11}=1+\cos ^{2} \alpha, \quad b_{12}=\frac{2 \beta_{T_{\|}}^{2}}{\pi|W(\xi)|^{2}} \frac{\sin ^{4} \alpha\left(1+2 \cos ^{2} \alpha\right)^{2}}{\left(1+\cos ^{2} \alpha\right)^{3}}, \\
\left.b_{s l}\right|_{s \geq 2}=\frac{\left(1+K_{s l} \cos \alpha\right)^{2}}{1+K_{s l}^{2}}, \quad K_{s l}=\frac{2 s \cos \alpha}{\sin ^{2} \alpha \pm \sqrt{\sin ^{4} \alpha+4 s^{2} \cos ^{2} \alpha}} .
\end{gathered}
$$

correspond to the case where the mode polarization is determined by the rarefied plasma (the vacuum birefringence effects are negligible). Here, the upper sign in the last formula corresponds to the extraordinary mode $(l=1)$ and the lower, to the ordinary one $(l=2)$, and $W(\xi)$ is the Kramp function.

The Landau-level populations are determined by impact transitions caused by particle collisions and radiative transitions due to interaction with cyclotron photons. Since the probability of radiative transitions at the first cyclotron harmonic is much higher than the higher-harmonic radiative transitions, the corresponding steady-state balance equations have the form (Zheleznyakov \& Litvinchuk 1984; Zheleznyakov \& Serber 1993; Serber 1999)

$$
(j+1)\left[\left(1+q_{1}\right) N_{j+1}-q_{1} N_{j}\right]-j\left[\left(1+q_{1}\right) N_{j}-q_{1} N_{j-1}\right]=\varepsilon\left(N_{j}-N_{j}^{(0)}\right),
$$

where $N_{j}^{(0)}=N\left(1-e^{-\hbar \omega_{B} / \kappa T_{\|}}\right) e^{-j \hbar \omega_{B} / \kappa T_{\mid}}$are the populations corresponding to a Boltzmann distribution with temperature $T_{\|}$, 


$$
q_{1}=\frac{4 \pi^{3} c^{2}}{\hbar \omega_{B}^{3}} \int_{-1}^{1} \frac{I_{11}(z, \xi, \alpha) \psi_{11}(\alpha)+\varpi(\xi) I_{12}(z, \xi, \alpha) \psi_{12}(\alpha)}{1+\varpi(\xi)} \mathrm{d} \cos \alpha
$$

is the photon occupation number at the first harmonic,

$$
\psi_{s l}(\alpha)=b_{s l}(\alpha) \sin ^{2 s-2} \alpha\left(2 \pi \int_{-1}^{1} b_{s l}(\alpha) \sin ^{2 s-2} \alpha \mathrm{d} \cos \alpha\right)^{-1}
$$

are the normalized angular patterns, and $\varpi(\xi)=\beta_{T_{\|}}^{2}|W(\xi)|^{-2}(147 / 16-28 / \pi)$. The left-hand side of Eq. (4) describes the spontaneous and stimulated cyclotron transitions at the first harmonic between level $j$ and levels $j \pm 1$. The right-hand side of this equation accounts for the variation in the population of the $j$ th level due to electron-proton collisions within the framework of the simplest relaxationtime approximation.

Using the generating-function technique, Serber $(1990,1999)$ solved analytically the infinite chain of coupled difference equations (4) and found the nonLTE transfer coefficients for cyclotron radiation of the type $l$ at any harmonic $s$ in the case where the electron distribution is determined by the first-harmonic radiation and collisions:

$$
\begin{gathered}
\eta_{s l}=\frac{\hbar \omega_{B}^{3} s^{3}}{8 \pi^{3} c^{2}} F_{s l} N \sum_{j=0}^{s} \frac{s !}{j !} \frac{\varepsilon q_{1}^{s-j} q_{0}^{j}}{(\varepsilon+j)(\varepsilon+j+1) \ldots(\varepsilon+s)} \\
\chi_{s l}=F_{s l} N \sum_{j=0}^{s} \frac{s !}{j !} \frac{\varepsilon\left[\left(1+q_{1}\right)^{s-j}\left(1+q_{0}\right)^{j}-q_{1}^{s-j} q_{0}^{j}\right]}{(\varepsilon+j)(\varepsilon+j+1) \ldots(\varepsilon+s)} \\
q_{0}=\left(e^{\hbar \omega_{B} / \kappa T_{\|}}-1\right)^{-1}=\frac{8 \pi^{3} c^{2}}{\hbar \omega_{B}^{3}} B_{\omega}^{\|}\left(\omega_{B}\right) .
\end{gathered}
$$

These transfer coefficients are valid both in quasi-classical and in quantized plasma for an arbitrary $\varepsilon$. If $\varepsilon \gg 1$, then, according to Eq. (4), the Landau-level populations correspond to the Boltzmann distribution with the temperature $T_{\perp}=T_{\|}$. In such a "collisional" plasma the electron distribution, formed by frequent electron collisions, is isotropic and independent of cyclotron radiation, so that the LTE is realized and the Kirchhoff law for the cyclotron radiation is valid. In the opposite limiting case of "collisionless" plasma where $\varepsilon \ll 1$, the electron distribution over the Landau levels is also Boltzmann, but it is formed by radiative cyclotron transitions at the first harmonic. Here, the temperature $T_{\perp}$ is equal to the brightness temperature of the cyclotron radiation at the first harmonic: $q_{1}=\left(e^{\hbar \omega_{B} / \kappa T_{\perp}}-1\right)^{-1}$, which is different at different points of the plasma due to the effects of radiation transfer.

Therefore, to calculate cyclotron radiation transfer under non-LTE conditions, one should first solve the transfer equations for the ordinary and extraordinary modes at the first harmonic with allowance for the relevant boundary conditions, determine the distribution of $q_{1}$ (or, equivalently, the temperature $T_{\perp}$ ) in the plasma, substitute the obtained distribution into Eqs. (5) and (6) for $s>1$, and, finally, solve the resulting transfer equations for higher harmonics. 
The transfer equations at the first harmonic are as follows (Zheleznyakov, Koryagin, \& Serber 1999):

$$
\begin{gathered}
\cos \alpha \frac{\partial I_{11}(\tau, \xi, \alpha)}{\partial \tau}=\frac{\phi(\xi) \Psi_{11}(\alpha)}{|\cos \alpha|}\left[I_{11}(\tau, \xi, \alpha)-S_{1}(\tau, \xi)\right], \\
\cos \alpha \frac{\partial I_{12}(\tau, \xi, \alpha)}{\partial \tau}=\frac{\phi(\xi) \varpi(\xi) \Psi_{12}(\alpha)}{|\cos \alpha|}\left[I_{12}(\tau, \xi, \alpha)-S_{1}(\tau, \xi)\right],
\end{gathered}
$$

where $S_{1}=\left(\hbar \omega_{B}^{3} / 8 \pi^{3} c^{2}\right) q_{1}$ is the source function, and

$$
\tau(z)=\frac{4 \sqrt{2} \pi^{2} e^{2} \phi(\xi) N(H-z)}{m c \beta_{T_{\|}} \omega_{B}}
$$

is the optical depth reckoned from the upper boundary of the slab. The total optical depth of the slab at the first harmonic is $\tau_{0}=\tau(0)$. The $z$ axis is directed vertically out, and $z=0$ corresponds to the lower boundary of the slab. Note that no radiation is incident on the slab from above. Moreover, the temperature of the WD surface is much lower than the slab temperature, so the influence of radiation incoming to the slab from below is negligible. Hence, we seek the solution of system (7) for zero boundary conditions.

We find the source function $S_{1}$ in the two-flux approximation for the angleaveraged intensities

$$
I_{l}^{(1)}=\int_{0}^{1} I_{1 l}(\tau, \xi, \alpha) \mathrm{d} \cos \alpha, \quad I_{l}^{(2)}=\int_{-1}^{0} I_{1 l}(\tau, \xi, \alpha) \mathrm{d} \cos \alpha .
$$

In terms of these quantities, we have

$$
S_{1}=\frac{1}{\varepsilon+1} \frac{I_{1}^{+}+\varpi I_{2}^{+}}{2[1+\varpi(\xi)]}+\frac{\varepsilon B_{\omega}^{\|}\left(\omega_{B}\right)}{\varepsilon+1},
$$

where $I_{l}^{+}=I_{l}^{(1)}+I_{l}^{(2)}$. With allowance for the fact that $\varpi \ll 1$, solution of system (7) in the two-flux approximation with zero boundary conditions yields

$$
\begin{gathered}
I_{1}^{+}(\tau)=\frac{2 \varepsilon B_{\omega}^{\|}\left(\omega_{B}\right)+\varpi I_{2}^{+}(\tau)}{\varepsilon+\varpi}\left[1-\frac{e^{-\lambda_{1}\left(\tau_{0}-\tau\right)}+e^{-\lambda_{1} \tau}}{1+\lambda_{1}-\left(1-\lambda_{1}\right) e^{-\lambda_{1} \tau_{0}}}\right], \\
I_{2}^{+}(\tau)=2 B_{\omega}^{\|}\left(\omega_{B}\right)\left[1-\frac{e^{-\varpi \lambda_{2}\left(\tau_{0}-\tau\right)}+e^{-\varpi \lambda_{2} \tau}}{1+\lambda_{2}-\left(1-\lambda_{2}\right) e^{-\varpi \lambda_{2} \tau_{0}}}\right],
\end{gathered}
$$

where $\lambda_{1}=\sqrt{(\varepsilon+\varpi) /(\varepsilon+1)}$ and $\lambda_{2}=\sqrt{\varepsilon /(\varepsilon+\varpi)}$.

The outgoing intensities are determined by the formal solutions of the corresponding transfer equations with allowance for zero boundary conditions:

$$
I_{s l}^{\text {out }}(\alpha)=\int_{0}^{\tau_{s l}(0)} S_{s} e^{-\tau_{s l}} \mathrm{~d} \tau_{s l}
$$

Here, $S_{s}=\eta_{s l} / \chi_{s l}$ and $\tau_{s l}(z)=\int_{z}^{H}\left(\chi_{s l}(\xi, \tau, \alpha) / \cos \alpha\right) \mathrm{d} z$ are the source function and the optical depth along the line of sight at the sth harmonic. According to Eqs. (5) and (6), these quantities are functions of $S_{1}$. We calculate $S_{s}, \tau_{s l}$, and then $I_{s l}^{\text {out }}$ numerically using Eqs. (8) and (9). 
Table 1. Results of interpretation of phase resolved cyclotron spectra

\begin{tabular}{lcc}
\hline Source parameters & HS 1023+3900 & HS 0922+1333 \\
\hline Plasma temperature, keV & $1.5 \mathrm{keV}$ & $1.5 \mathrm{keV}$ \\
Relative size $\Theta_{*}=r / R_{*}$ of the spot & 0.1 & 0.1 \\
Magnetic field $B$ (main/secondary pole) & $60 / 68 \mathrm{MG}$ & $66 \mathrm{MG}$ \\
$\begin{array}{l}\text { Total optical depth at the 1st harmonic } \\
\text { (main/secondary pole) }\end{array}$ & $10^{7} / 5 \times 10^{4}$ & $10^{9}$ \\
Angle between the magnetic field and the & & $20^{\circ}$ \\
WD rotation axis & & $75^{\circ}$ \\
Angle between the line of sight and the & $86^{\circ} / 88^{\circ}$ & $25^{\circ}$ \\
WD rotation axis (main/secondary pole) & \\
\hline \hline
\end{tabular}

\section{Results and discussion}

The results of interpretation of the phase-resolved cyclotron spectra of low accretion rate polars HS $1023+3900$ and HS $0922+1333$ are given in Table 1 . Note that our model makes it possible to reproduce the two-hump temporal profiles of the secondary-pole 3rd harmonic for HS $1023+3900$ and the main-pole 4 th harmonic for HS $0922+\mathbf{1 3 3 3}$.

The features of the considered model of non-LTE cyclotron radiation transfer can be explained on the basis of the Barbier-Eddington relation according to which $\left.I_{s l}^{\text {out }} \simeq S_{s}\right|_{\tau_{s l} \simeq 1}$. In the case of LTE where the source functions $S_{s} \simeq B_{\omega}\left(s \omega_{B}\right)$ are constant in an isothermal slab, the outgoing intensities for any $s, l$, and $\alpha$ are close to $B_{\omega}(\omega)$ unless the slab is optically thin along the line of sight. Thus, it is hardly possible to obtain two-hump temporal profiles of higher-harmonic cyclotron features without special assumptions on the emitting-region geometry. On the contrary, in our non-LTE model, $S_{1}$ is an even function with respect to the slab center, so that $S_{1}\left(\tau_{0} / 2\right) \simeq B_{\omega}\left(\omega_{B}\right)$ and $S_{1}(0)=S_{1}\left(\tau_{0}\right) \ll B_{\omega}\left(\omega_{B}\right)$ at the slab boundaries. Correspondingly, the source functions $S_{s}$ are also even with respect to the slab center. These functions have the maxima $S_{s} \simeq B_{\omega}\left(s \omega_{B}\right)$ for $\tau_{0} / 2$ and decrease to values much lower than $B_{\omega}\left(s \omega_{B}\right)$ near the slab boundaries. As for the optical depths $\tau_{s l}$ for $s>1$, these quantities increase monotonically from 0 to infinity as $\alpha$ varies from 0 to $\pi / 2$.

Let us now explain qualitatively the temporal behavior of the main-pole 3rd and 4th harmonics in the phase-resolved spectrum of HS $0922+1333$ taking into account the above-mentioned properties of $S_{s}$ and $\tau_{s l}$. The angle $\alpha$ is equal to $\pi / 2$ at the egress phase. Then this angle decreases due to the WD rotation, reach some minimum value $\alpha_{0} \simeq 50^{\circ}$, and increases again reaching $\pi / 2$ at the ingress phase. The observed spectral flux of cyclotron radiation at the $s$ th harmonic is proportional to $I_{s l}^{\text {out }} \cos \alpha$. Under conditions of the main pole of HS $0922+1333$, the 3rd harmonic remains optically thick for $\alpha_{0}<\alpha<\pi / 2$. As $\alpha$ varies from $\pi / 2$ to $\alpha_{0}$, the point at which $\tau_{3 l} \simeq 1$ shifts from the upper boundary of the slab where $S_{3} \ll B_{\omega}\left(3 \omega_{B}\right)$ to inner regions of the slab where the radiation is thermalized and $S_{3} \simeq B_{\omega}\left(3 \omega_{B}\right)$. As a result, the phase dependence of the observed flux at the 3rd harmonic shows single flat-top maximum. The flat part of this dependence 
corresponds to those values of $\alpha$ for which the point $\tau_{3 l} \simeq 1$ remains into the region of thermalized radiation. On the contrary, the 4 th harmonic becomes optically thin for $\alpha_{0}<\alpha<\alpha_{*}$, where $\alpha_{*}<\pi / 2$ As a result, the observed flux at this harmonic becomes very weak for this range of $\alpha$, and the corresponding phase dependence shows two humps and a minimum in between. The center of this minimum corresponds to $\alpha=\alpha_{0}$.

Acknowledgments. This work is supported by the Russian Foundation for Basic Research (project No. 02-02-16236), the Council for the State Support of Leading Scientific Schools (grant No. 00-15-96674), the program "Nonstationary Phenomena in Astrophysics" of the Russian Academy of Sciences, and the "Astronomy" program of the Russian Ministry of Industry, Science, and Technologies. A.S. is sincerely grateful to the IAU and the Organizing Committee of this colloquium for a travel grant supporting his participation in the meeting.

\section{References}

Reimers, D., Hagen, H.-J., \& Hopp, U. 1999, A\&A, 343, L57

Reimers, D., \& Hagen, H.-J. 2000, $A \& A$, 358, L45

Schwope, A., Brunner, H., Hambaryan, V. \& Schwarz, R., 2002, in The Physics of Cataclysmic Variables and Related Objects, eds B. T. Gänsicke, K. Beuermann, \& K. Reinsch (San Francisco: ASP), ASP Conf. Ser. 261, 102

Serber, A. V. 1990, Soviet Ast., 34, 291

Serber, A. V. 1999, Radiophys. Quantum Electron., 42, 911

Zheleznyakov, V. V. 1983, Ap\&SS, 97, 229

Zheleznyakov, V. V. 1996, Radiation in Astrophysical Plasmas (Kluwer)

Zheleznyakov, V.V., Koryagin, S.A. \& Serber, A. V. 1999, AstL, 25, 445

Zheleznyakov, V. V. \& Litvinchuk, A. A. 1984, Ap\&SS, 105, 73

Zheleznyakov, V. V. \& Litvinchuk, A. A. 1987, Soviet Ast., 31, 159

Zheleznyakov, V. V. \& Serber, A. V. 1993, Soviet Ast., 37, 507 